This article has been accepted for publication in a future issue of this journal, but has not been fully edited.

Content may change prior to final publication in an issue of the journal. To cite the paper please use the doi provided on the Digital Library page.

\title{
Rapid Multi-Objective Design Optimization of Switched Reluctance Motors Exploiting Magnetic Flux Tubes
}

\author{
Aleksas Stuikys *, Jan Sykulski \\ Department of Electronics and Computer Science, University of Southampton, Southampton, UK \\ *A.Stuikys@soton.ac.uk
}

\begin{abstract}
The magnetic design of Switched Reluctance (SR) motors is inherently a hierarchical process. The design cycle progresses through distinct stages where the accuracy improves but computing times increase greatly, thus it often becomes impractical to furnish extensive multi-objective optimization required to accomplish the optimal design. In order to enable rapid and accurate optimization of SR motors an improved reduced-order computational method of flux tubes is implemented to complement and practically replace the time consuming 2D finite element based magnetic analysis. The paper demonstrates how the use of the improved flux tubes approach to evaluate objective functions results in substantially faster while still accurate multi-objective optimization of SR motors.
\end{abstract}

\section{Introduction}

Switched Reluctance (SR) machines are often regarded as having the simplest mechanical design [1]-[3] compared to other conventional electric machines, such as permanent magnet dc, synchronous reluctance or induction, as they are brushless and have no permanent magnets (PM) or windings on the rotor. These design features make SR machines well suited to a wide range of applications where variable speed operation is required, such as general traction or pump drives. Moreover, the mechanical robustness of SR machines offers cheaper maintenance and better tolerance to harsh environments in which other types of machines cannot operate.

Since SR motors are capable of operating in both constant torque and constant power regimes over a wide speed range [4], [5], this operating feature also makes them suitable for the automotive propulsion applications, where the absence of permanent magnets is considered necessary since this industry is very cost sensitive and insists on immunity from unforeseen material price fluctuations, as experienced by PM materials [6]. However, the absence of PM parts makes the SR machines much more difficult to control [7] due to their nonlinear torque-per-ampere characteristic. This operational nonlinearity is a result of the nonlinear magnetic characteristic as the machine's magnetic circuit becomes heavily saturated even during steady-state operation [8].

Considering that SR machines are very nonlinear, they require complex analysis and robust design procedures to adequately predict their performance. Until now a rather limited success has been achieved in terms of accurate analytical design procedures for SR machines [9], [10] to provide satisfactory quantification of the machine performance. Most of the magnetic analysis and design is nowadays performed by numerical simulations based on a finite element method (FEM) in order to capture the detailed shape and allow for magnetic nonlinearity. The FEM based solutions can be accurate; however, they are time consuming and lack the intuitive insight into the cause-and-effect relationships between numerous machine design parameters. Moreover, the FEM solutions give only part of the information needed to fully describe the performance; this necessitates extensive post processing of the results using specialist software. Lengthy FEM solver based design computations resulted in the previous FEM based Differential Evolution (DE) optimization attempts to be rather restricted in terms of the number of objective function calls, with most such investigations taking more than 10 hours to complete and the number of design points explored being limited to about 200 [11] - with simultaneous three objectives to be optimized this number of design points appears to be insufficient. Furthermore, the same difficulty is encountered with the use of Design of Experiments (DoE) based optimizations as the number of objective function calls based on FEM solver is large even if the number of design objectives is less than 3 [12]. Single design-objective based optimizations have shown some success in the past for the machines which exhibited good initial design configurations, i.e. were known to be good designs prior to the single-objective optimization, such as torque ripple minimization [13]. On the other hand, an optimization approach using only a small number of machine design parameters and design objectives is unlikely to result in an optimal design, as a large design space is normally needed to avoid local optima. These conflicting requirements naturally lead to the concept of a hierarchical design as depicted by the inverted pyramid of Fig. 1.

The hierarchical design process summarized in Fig. 1 indicates that at each subsequent optimization step the design space of a particular electromechanical device may be reduced as the confidence in the predicted performance grows following the outcome of the previous design stage. The clear distinctions between the different design paradigms in the pyramid emphasize that accuracy generally improves as we use more elaborate analysis tools at the expense, however, of significantly increased computational effort. Thus there is a need for both approximate (but fast) modelling tools at the early stages of the design, to explore the large design space, while homing in on a particular area identified as the likely location of the global optimum allows for the use of more time consuming simulation tools as fewer design points need to be considered. In the context of electromechanical devices this stage is often further split 
This article has been accepted for publication in a future issue of this journal, but has not been fully edited.

Content may change prior to final publication in an issue of the journal. To cite the paper please use the doi provided on the Digital Library page.

into preliminary 2D steady state FEM simulations followed by the most computationally intensive transient 3D FEM solutions. The challenge here is to ensure a smooth transition between the models without the loss of important information, especially that stepping 'back' in the modelling (from more to less accurate models) may sometimes be required if an area outside the currently localized optimum needs to be explored further. Associated with this is the issue of selecting the most appropriate optimization algorithms, as the simple but fast models may be run many times while with the FEM based simulations we aim at reducing the number of objective function calls. Thus there may be several internal communication links between the different levels in the pyramid. The design of a SR motor falls neatly into the paradigm discussed above where a careful balance needs to be achieved between the speed of computation and accuracy of the solution.

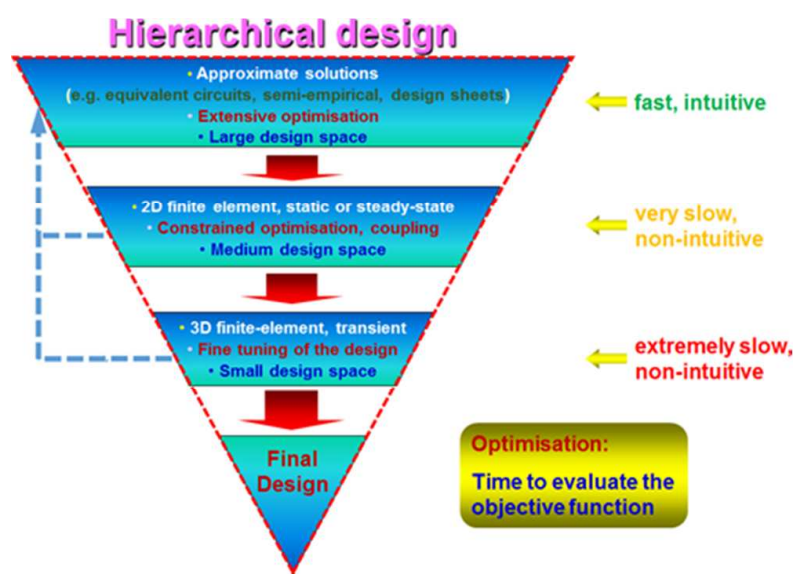

Fig. 1. The concept of a hierarchical design of electromechanical devices.

This paper proposes to improve the efficiency of analysis and design optimization of the radial flux SR machine by adapting the improved reduced order computational method of flux tubes. The flux tube method which has already been successfully applied to translating SR machines as reported in [14] and [15] - complements and enhances the hierarchical design paradigm of Fig. 1 by reducing the need for hard choice between solution time and accuracy early on in the design process, while still allowing to arrive at a near-optimal design. Therefore the importance of the intermediate stage (one which is very slow and nonintuitive) in the hierarchical process of Fig. 1 is diminished since the enhanced accuracy of the approximate but fast solution based on flux tubes can be directly utilized in the final design stage with substantial savings in computational resources.

The reduced order computational method of flux tubes is a well-established technique [16]; however, its application to the design of SR machines has not been pursued and in the past the design process relied heavily on the manual work of the designer [3], [17]-[19]. In this paper an improved and extended version of the flux tube method is proposed whereby the original flux tube modelling is combined with tubes-and-slices (TAS) techniques [20], resulting in a substantial reduction of manual work of an analyst or a designer, while improving the computing speed and making the accuracy comparable to 2D FEM. It is argued that this approach makes it particularly appropriate in everyday engineering practice in design offices.

The reminder of the paper is organized as follows. In Section 2 the application of the improved flux tube method, with emphasis on computing speed and accuracy, is illustrated using an example of a prototype SR motor with published measured performance data. In Section 3 a detailed discussion follows related to the robustness of the improved flux tube method. Section 4 describes an application of the flux tube method to a multi-objective optimization of the SR motor for automotive traction application. In Section 5 the proposed SR machine design optimization cycle is discussed, Fig. 12, and demonstrated how it complements and enhances the conventional design process of Fig. 1. Finally, Section 6 contains the conclusions and suggested future research direction in the field of rapid multi-objective optimization of SR machines and other electric machines using the flux tubes.

\section{THE FLUX TUBE MODELLING APPROACH}

In this section the proposed improved flux tube method is applied to a $50 \mathrm{~kW}$ radial flux $18 / 12$ SR motor intended for automotive traction application, as published in [4], where most of the design and optimization was achieved using FEM. A prototype was built and a full dynamometer test performed in order to measure its torque output over a wide speed range. The machine was subsequently redesigned to improve its performance compared to the initial version. The relevant data for both machines (SRM1 and SRM2) is listed in Table 1.

Table 1 SR Motor Design Variables and Test Results (adapted from [4]).

\begin{tabular}{ccc}
\hline Design Parameters & SRM1 & SRM2 \\
\hline Outer diameter [mm] & 269 & 269 \\
Stack length [mm] & 135 & 135 \\
Air gap length [mm] & 0.5 & 0.5 \\
Iron core material & 10JNEX900 & 10JNEX900 \\
Wire diameter [mm] & 0.95 & 0.6 \\
Wire turns [turns] & 13 & 17 \\
Wire parallel turns [turns] & 13 & 22 \\
Slot fill factor [\%] & 54.1 & 57.0 \\
Current, peak [A] & 380 & 320 \\
Max. current density & $24(24)$ & $33(33)$ \\
[A/mm ${ }^{2}$ ] & & \\
RMS current (@1200rpm) & $241(240)$ & $204(206)$ \\
[Arms] & & \\
Max Torque (@1200rpm) & $354(340)$ & $415(400.4)$ \\
[Nm] & 3 & 3 \\
Number of phases & &
\end{tabular}

( ) denote test results

As the geometries and other data for both SR machines are known, including material properties and electric loading, a 2D FEM analysis of the SRM2 design was performed in order to visualize the magnetic flux distribution within the magnetic circuit of the machine. Fig. 2 illustrates a general solution for the SRM2 design at a peak current as specified in Table 1. The flux tube modelling approach will now be explained in detail. 
This article has been accepted for publication in a future issue of this journal, but has not been fully edited.

Content may change prior to final publication in an issue of the journal. To cite the paper please use the doi provided on the Digital Library page.

The flux tube modelling of the SR machine starts with a general inspection of the 2D FEM solution, as illustrated in Fig. 2, and in particular inspection of the flux function distributions within the magnetic circuit. The equipotential lines - along which the magnetic field density is constant, or approximately constant - are chosen in such a way so that the complete magnetic circuit can be fully subdivided along its length. Subsequently, the magnetic circuit is segmented into the identified equipotential slices as shown in Fig. 3, while taking full advantage of the machine symmetry.

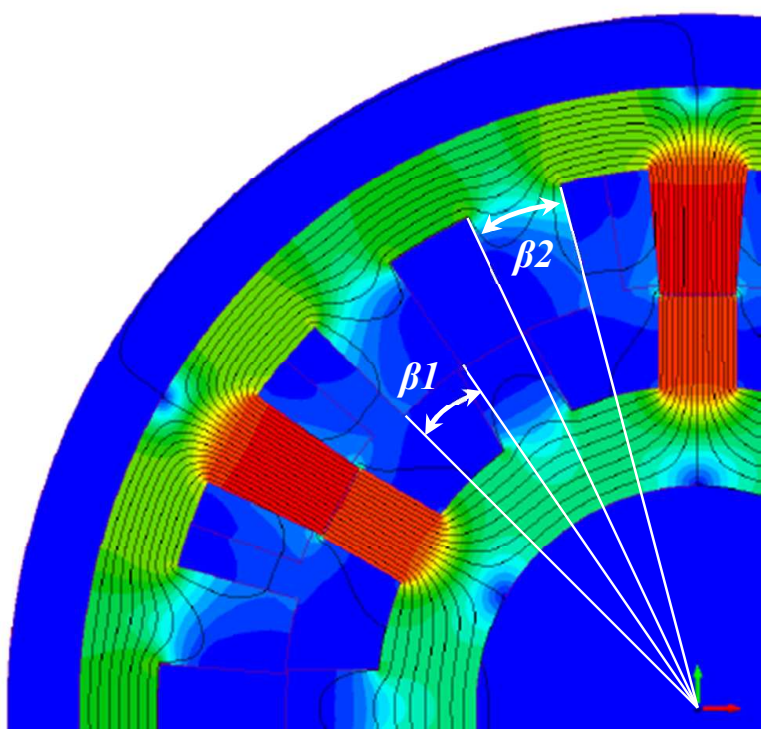

Fig. 2. 2D FEM solution for the 18/12 three phase SRM2 design at the aligned rotor position.

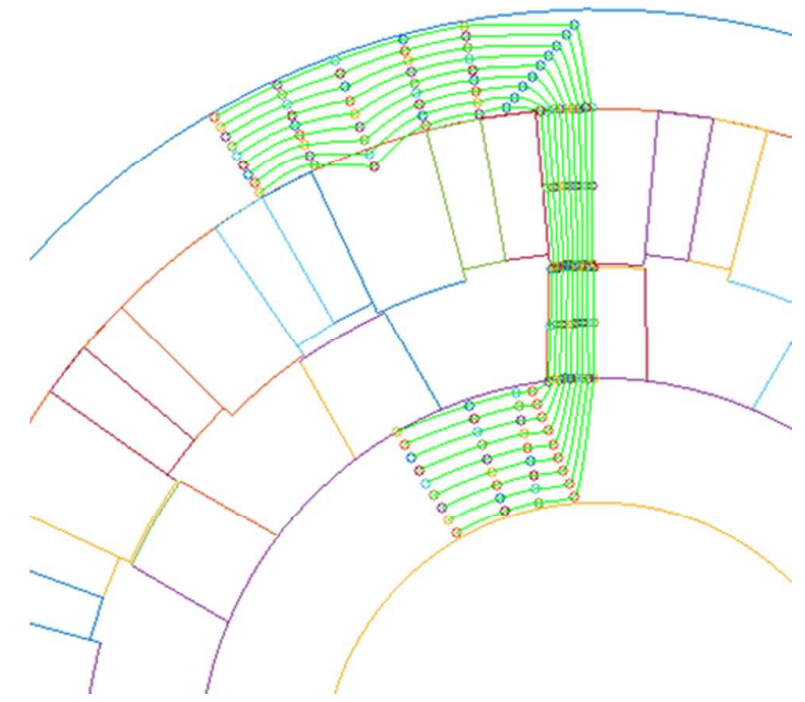

Fig. 3. Subdivision of the SRM2 magnetic circuit into equipotential slices at the aligned rotor position using flux tubes methodology.

The equipotential slices in Fig. 3 are fixed to the given machine geometry by associating the slices with the machine geometric parameters and therefore are free to vary as the machine geometry is varied.
Once the subdivision into the equipotential slices is complete the equipotential slices are mapped into rows of coordinate points between which the smooth and continuous cubic-spline interpolations are performed automatically in order to approximate, as closely as possible, the true magnetic flux function lines, as those in Fig. 2. Thus the resulting smooth line segments in Fig. 3 along the path of the magnetic circuit represent magnetic flux tubes of equal flux inside them. The knowledge of the constructed flux tube geometric properties, as well as material properties in which they occur, enables accurate estimation of the flux linkage functions for a particular SR machine geometry and prescribed electric loading. The flux linkage function for both the aligned and unaligned rotor positions of the SR machine can be defined analytically as

$$
\psi=\frac{N^{2} \cdot I}{R} .
$$

where $N$ is the number of conductor turns, $I$ is the electric current and $R$ is the magnetic reluctance of the magnetic circuit in which the flux tubes are established [16]. Therefore the numerator in (1) is a known quantity as the number of turns and the current are both given. Consequently the only unknown value in (1) is the magnetic reluctance of the magnetic circuit which - in the most general case - is a nonlinear quantity and varies as a function of the current. It transpires that the fitted flux tubes in Figs. 3 and 6 give close estimates of the reluctance values $R$ for both rotor positions. We will demonstrate how the magnetic reluctance is estimated and the reasons behind the numerical robustness of the flux tube method in Section 3. Using (1) the flux linkages are found for both rotor positions for the two SR machine designs considered in Table 1 and are shown in Fig. 4.

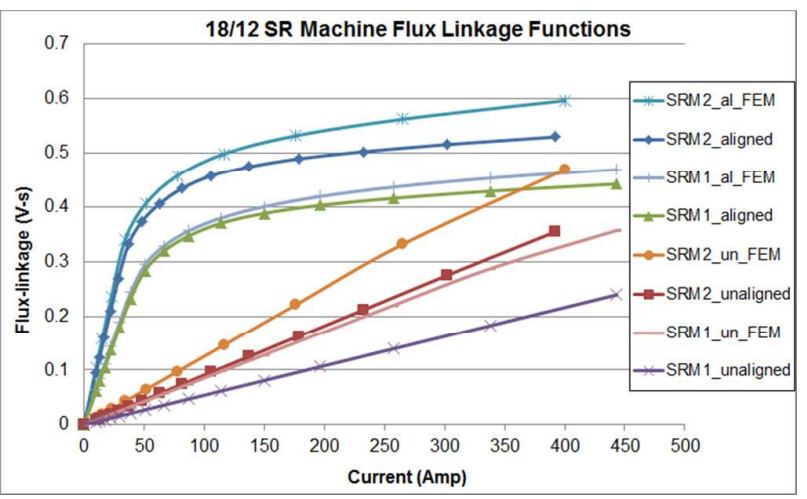

Fig. 4. The flux linkage functions for the SRM1 and SRM2 SR machine designs obtained with the flux tubes method and from the 2D FEM.

The unaligned flux linkage function of Fig. 4 for the SR machine may be estimated by constructing a flux tube model for the unaligned magnetic circuit, as shown in Fig. 6. A similar as before visual inspection of the FEM field plot of Fig. 5 is undertaken first in order to locate approximate equipotential magnetic field density contours which serve as equipotential slices of the magnetic circuit. Once such equipotential magnetic field density slices are found, 
This article has been accepted for publication in a future issue of this journal, but has not been fully edited.

Content may change prior to final publication in an issue of the journal. To cite the paper please use the doi provided on the Digital Library page.

constructed and converted into coordinate points, the automatic generation of flux tubes for the unaligned rotor position can be performed as shown in Fig. 6. Next, the total reluctance $R$ of the flux tubes is found from the geometric information about the flux tubes and is used in (1) to find the flux linkage of the unaligned rotor position as in Fig. 4.

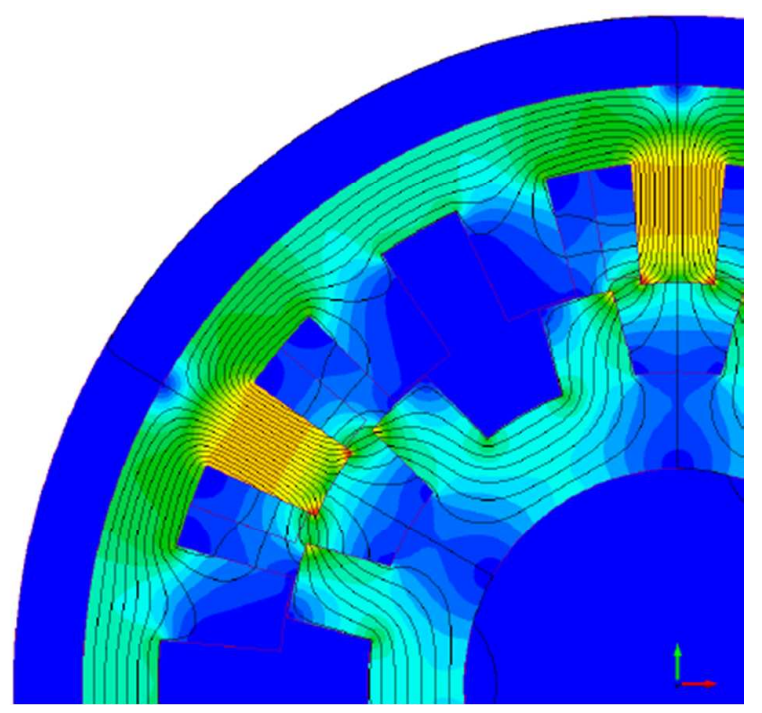

Fig. 5. 2D FEM solution for the 18/12 three phase SRM2 design at the unaligned rotor position.

The visual comparison of flux function distributions in Figs. 5 and 6 reveals that in Fig. 6 the flux tubes are not exactly replicated, but only approximately. However, the numerical comparison of the results obtained from the relevant distributions, as presented in Fig. 4, indicates that the flux tube method gives values sufficiently close to the associated 2D FEM results.

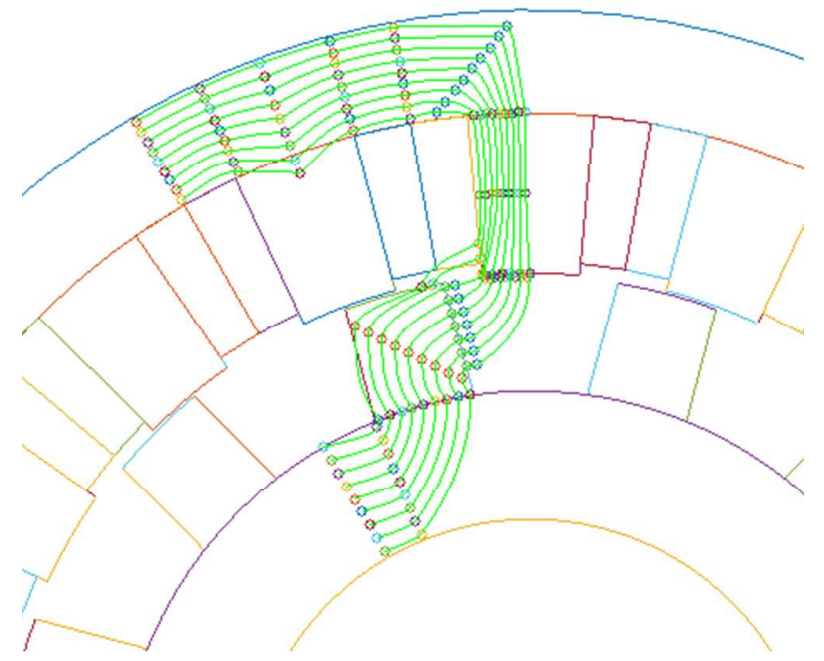

Fig. 6. Subdivision of the SRM2 magnetic circuit into equipotential slices at the unaligned rotor position using flux tube methodology.

It should be noted at this point that the flux tube based flux linkage estimation took $1 \mathrm{~s}$ compared to $45 \mathrm{~s}$ in the case of the 2D FEM aligned case which was the faster of the two FEM cases, not taking into account the FEM model geometry preparation and solver setup time. Fig. 4 indicates that both of the SRM2 machine flux linkage functions are underestimated compared to the FEM based solutions. The aligned flux linkage function of flux tubes is underestimated by around $11 \%$ and the unaligned by $24 \%$ at the $400 \mathrm{~A}$ current level which is above the peak current limit of the machines as specified in Table 1. The underestimation of the flux tube based analysis results is due to the fact that the flux tube method constrains the flux tube paths which are then fixed for all operating current levels of the machine, whereas in the 2D FEM based solution the flux tubes are positioned in such a way as to minimize the path reluctance for each current level. In other words, the leakage flux was not incorporated into the flux tube model. The flux linkage value discrepancies in Fig. 4 may be attributed to the absence of the leakage flux in the flux tube model. As a partial remedy, to account for the discrepancies in the flux linkage values quoted above and in Fig. 4, two calibration parameters (aligned and unaligned) were introduced, both proportional to the errors, in order to minimize the likely numerical errors from the subsequent flux tubes based simulations.

Having obtained the flux linkage functions for the SRM2 machine design, as detailed in Table 1, the complete flux linkage map of the machine can be efficiently and accurately constructed using the gauge curve fitting techniques as detailed in [1]. Therefore it is not necessary to model the intermediate rotor position flux functions. Once the complete flux linkage map of the SR motor is available, the speed-torque and output power characteristics of the machine can be obtained, given that the operating voltage, current and firing angles are known. The characteristics analysis is performed as a flux-tubes post-processing step and thus the post-processing times are not measured since they do not depend on the magnetic field analysis times. In order to compare the result from the flux tube method with the measurements cited in [4] the SRM2 motor performance was evaluated at a rated machine speed of $1200 \mathrm{rpm}$. The flux tube based flux linkage map gives rise to the torque value of $420 \mathrm{Nm}$ at the rated machine speed, an overestimate by $5 \%$ compared with the measurement, but very close to the result from the FEM analysis. Moreover, the estimated RMS value of the current for the SRM2 machine at the rated speed is $220 \mathrm{~A}$, a $7 \%$ overestimate compared with the measurement. Overall the very fast estimates based on the flux tube method appear to be close, from the practical point of view, to the experimental results and comparable to those obtained from the much more computationally expensive 2D FEM.

\section{Accuracy and Robustness of the Flux Tube Method}

The flux tube method was first formulated analytically in [16]; it is possible to derive equations governing the accuracy of the numerical approach based on this method. A detailed account of the derivations may be found in [15], a summary is given below.

Assume a system of tubes arranged in parallel to each other to occupy an arbitrary shaped 3D space as in Fig. 7. Let each such tube contain a magnetic flux $\phi$ due to a difference in magnetic potentials $V_{a}$ and $V_{b}$ at a known 
This article has been accepted for publication in a future issue of this journal, but has not been fully edited.

Content may change prior to final publication in an issue of the journal. To cite the paper please use the doi provided on the Digital Library page.

distance $l_{a b}$ apart. If the flux tubes are subdivided into slices of arbitrary length along their entire length (between the nodes $V_{a}$ and $V_{b}$ ) the cross-sectional area of each such slice of each tube can be replaced by an average

$$
A_{j} \leftarrow \frac{1}{2}\left(A_{j}+A_{j+1}\right), \quad j=1,2, \ldots, n
$$

where the arrow indicates a substitution. Magnetic flux will of course exist in both the magnetic parts of the machine (iron) and in the air gap, the latter usually the more important from the point of view of the total $\mathrm{mmf}$ requirements. In terms of error analysis it is helpful to consider the air gap and iron parts separately as the final expressions are somewhat different.

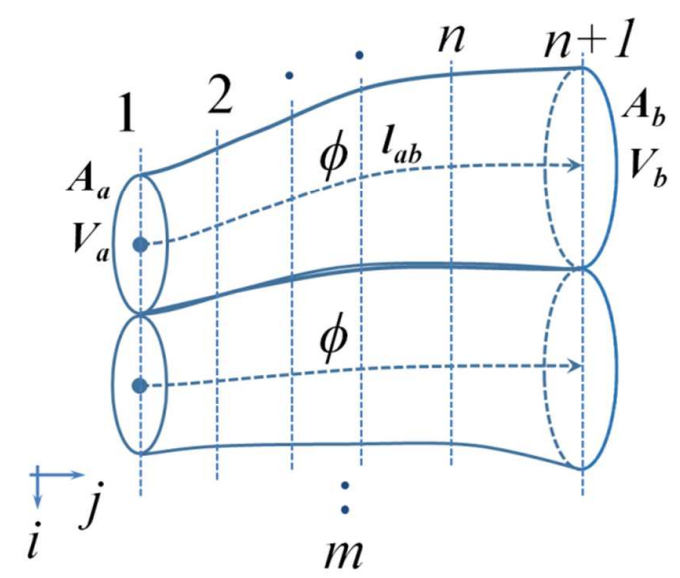

Fig. 7. A system of magnetic flux tubes arranged in parallel.

\subsection{Flux Tubes in the Air Gap}

The magnetic reluctance of each tube is approximated by

$$
R_{a b}=r_{t}=\sum_{j=1}^{n} \frac{l_{j}}{\mu_{0} \cdot A_{j}} .
$$

where $l_{j}$ is the length of each component sub-tube (with the ends defined by the appropriate slices). The higher the number of subdivisions $(n)$ the better chance to approach the true value of the reluctance $R$ as in (1), providing the tube paths are positioned correctly, with tubes arranged in parallel. Following this line of reasoning it was shown in [15] that the total reluctance of the system of tubes in air, as in Fig. 7, will be given by

$$
\frac{1}{R_{\text {parallel }}^{\prime}}=\sum_{i=1}^{m}\left(\sum_{j=1}^{n} \frac{l_{i j}}{\mu_{0} \cdot A_{i j}}\right)^{-1} \times \frac{(1+\alpha)}{(1+\lambda)}
$$

where $\alpha$ is an average error for the flux tube areas and $\lambda$ is an average error for the flux tube lengths, both of which can be numerically positive, negative or zero. The meanings of these errors may be explained with the help of Fig. 8, where a flux tube will be, in most cases, only approximately close to the shape of the 'true' tube (i.e. obtained from a 2D FEM). This may be captured by the following expressions

$$
A_{j} \cdot \varepsilon_{\text {A.av }}=A_{j}+A_{j} \cdot \alpha ; \quad l_{j} \cdot \varepsilon_{l \cdot a v}=l_{j}+l_{j} \cdot \lambda .
$$

where the relevant errors $\varepsilon$ have been averaged.

A substitution into (3) and application of the basic formula for a parallel connection of reluctances ultimately leads to the expression (4); as already mentioned the full derivation may be found in [15].

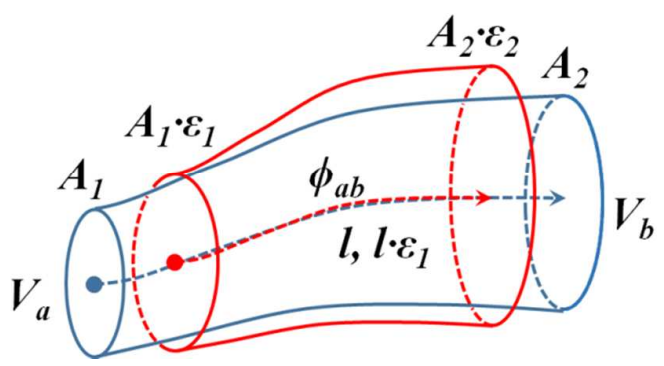

Fig. 8. An approximation of a tube introducing the errors in length and area.

Considering the implications of (4) it emerges that if both errors were positive, or both negative, at the same time, the resultant error would benefit from some cancellation effect, as it is the ratio of the two errors that matters. The worst case scenario will occur when the areas are overestimated and the lengths are underestimated, or vice versa. The result in (4) also indicates that the errors will not affect the resultant value of the reluctance dramatically as even if the area and the length errors were both around $10 \%$ and of opposite sign, the overall error in the reluctance value would be limited to around $20 \%$. Moreover, such an error would occur only if all the modelled flux tubes experienced the worst case scenario simultaneously.

\subsection{Iron Circuit Flux Tubes}

A similar argument may be applied to the system of flux tubes in the magnetic parts of the machine resulting in the expression

$$
R_{\text {parallel }}^{\prime}=\left(\sum_{i=1}^{m}\left(\sum_{j=1}^{n} \frac{H_{i j} \cdot l_{i j}}{B_{i j} \cdot A_{i j}}\right)^{-1}\right)^{-1} \cdot(1+\pi) \cdot(1+\lambda) .
$$

where $\pi$ denotes an error introduced due to the uncertainties in the magnetization data of the magnetically nonlinear material [15], which can be in the region of $\pm 10 \%$ as typically found in laboratory measurements and $\lambda$ denotes an average error for the flux tube lengths, as in (5).

The repercussions of (6) are somewhat different than for the tubes in the air gap, as encapsulated by (4), as this time the cancellation of errors will occur when the component errors are of opposite sign. At the same time it transpires that the area error $\alpha$ is no longer important, whereas the error in magnetization data needs to be considered. The worst case scenario occurs when both errors in (6) are of the same sign.

Following the above discussion it is now possible to explain why the flux linkage functions in Fig. 4 - obtained from the approximate distributions in Figs. 3 and $6-$ give results close to the 2D FEM analysis. As the local errors given in (5) for the modelled tubes will vary from point to 
This article has been accepted for publication in a future issue of this journal, but has not been fully edited.

Content may change prior to final publication in an issue of the journal. To cite the paper please use the doi provided on the Digital Library page.

point in the magnetic circuit, the total error is likely to be small thanks to the mentioned cancellation effects in (4) and (6). This leads to an encouraging observation regarding the improved flux tube formulation that the method does not require any particular calibration and will perform satisfactorily in practical situations. To ensure the accuracy the number of slices $n$ in (2) should be kept at a reasonable level, depending on the geometry of the magnetic circuit. The robustness of the flux tube method is therefore assured.

\section{MULTI-OBJECTIVE OPTIMIZATION USING FLUX TUBES}

The analysis of the SR machine based on flux tubes has been shown to be fast and accurate for practical purposes, making the method an ideal choice for rapid initial optimization. To illustrate the optimization process the SRM2 machine from Table 1 had been selected for further improvements while the design constraints were kept fixed as originally imposed in [4]. The design variables with associated ranges and constraints for the multi-objective optimization undertaken are shown in Table 2.

Table 2 Parameters for the 18/12 SR Machine Optimization

\begin{tabular}{lcc}
\hline Design Parameters & Initial Value & Value Range \\
\hline Stator Outer Diameter & 269 & 269 \\
Back Iron Thickness & $17.25 \mathrm{~mm}$ & $5-25 \mathrm{~mm}$ \\
Rotor Pole Height & $19.5 \mathrm{~mm}$ & $10-30 \mathrm{~mm}$ \\
Stator Pole Height & $27 \mathrm{~mm}$ & $10-50 \mathrm{~mm}$ \\
Stator Pole Angle, $\beta 1$ & 11 degrees & $6-12$ degrees \\
The Pole Root Angle, $\beta 2$ & 11 degrees & $6-12$ degrees \\
Shaft Diameter & $100 \mathrm{~mm}$ & $80-160 \mathrm{~mm}$ \\
Wire Turns & $17 \mathrm{turns}$ & $5-50 \mathrm{turns}$ \\
Stack Thickness & $135 \mathrm{~mm}$ & $135 \mathrm{~mm}$ \\
Air Gap Length & $0.5 \mathrm{~mm}$ & $0.5 \mathrm{~mm}$ \\
Number of Phases & 3 & 3 \\
Peak Phase Current & $320 \mathrm{~A}$ & $320 \mathrm{~A}$ \\
Supply DC Voltage & $500 \mathrm{~V}$ & $500 \mathrm{~V}$ \\
\hline
\end{tabular}

In the table the stator pole angles are as defined in Fig. 2 whereas the rotor pole angle was equal to $\beta 1$.

Given that the flux tube method takes only $3 \mathrm{~s}$ to construct the complete flux linkage map of the investigated SRM2 SR machine, and noting that the number of design variables is quite high, the evolutionary design optimization routine based on Genetic Algorithm (GA) [21] was considered the best choice since the algorithm directly benefits from accurate and fast-to-evaluate objective functions as provided by the flux tube functions. The capability to perform constrained multi-objective optimization was a further incentive to select GA for this task.

Three objective functions for the SR machine were defined. The first objective was to maximize the average torque at a rated machine speed of $1200 \mathrm{rpm}$, as originally specified in [4]. Secondly, the speed range over which rated constant power could be sustained by the machine was also to be maximized; this objective function is measured in units of $\mathrm{kW} \times \mathrm{rpm}$. Finally, the third objective function was selected to maximize the machine efficiency at the rated speed of 1200rpm.

The GA based constrained multi-objective optimization routine was configured as follows. The population size of 100 individuals was chosen and the number of generations set to a value of 30 . Therefore in total 3,200 individuals were generated in order to optimize the three simultaneous objectives, for the seven design variables and subject to six constraints, as detailed in Table 2 . The GA solver was passed an 'elitism score' of 30\% - effectively limiting the count of elite parents (individuals) to $30 \%$ of the total population in a given generation. The elitism score prevents too large a number of suboptimal machine designs from being carried over into subsequent generations [21].

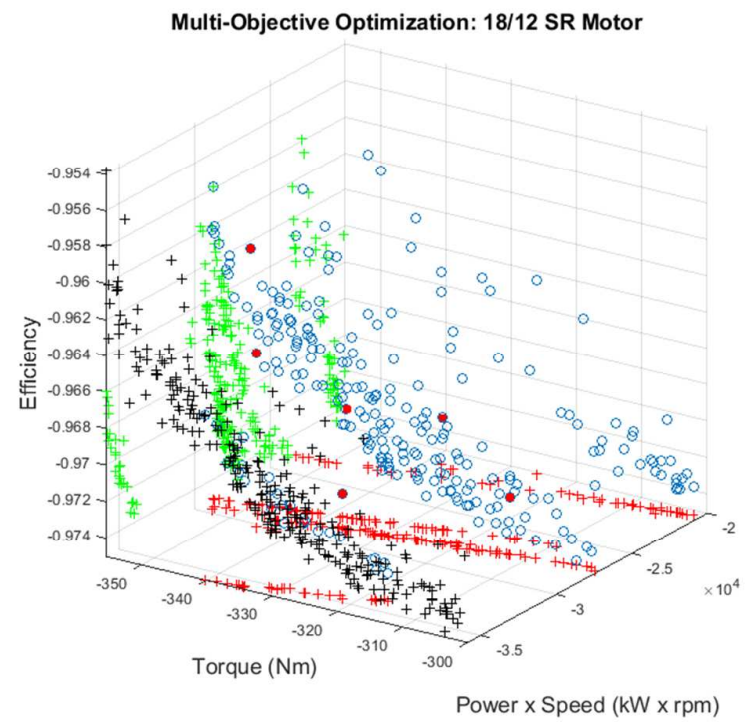

Fig. 9. 3D scatter plot of the three objective function design points (circles) and the projections of pareto fronts onto each plane (plus markers).

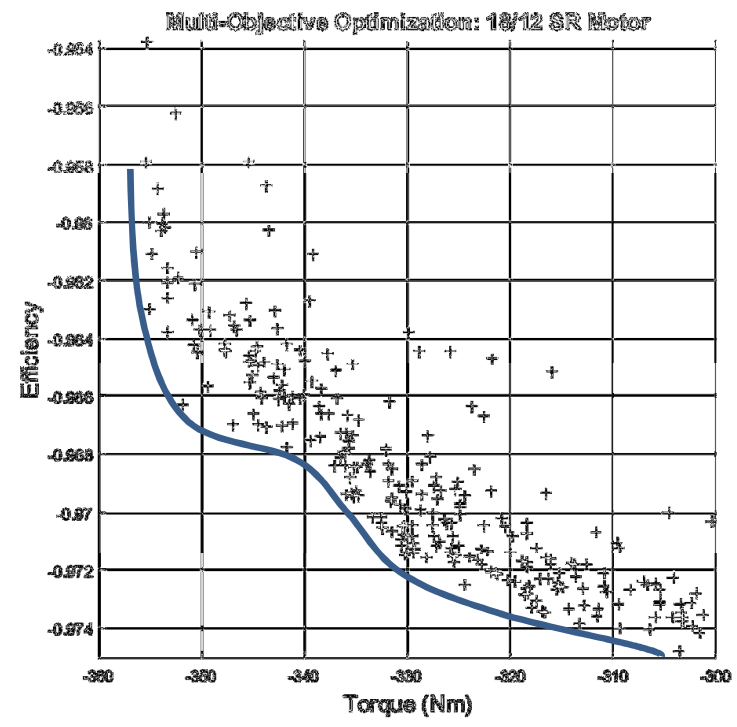

Fig. 10. 2D plot of the two objective functions with the approximate projection (solid line) of $3 D$ pareto front onto the plane.

Once the optimization task, using the GA, was complete the optimized three objectives for the 18/12 SR machine were simultaneously visualized as a $3 \mathrm{D}$ scatter plot 
This article has been accepted for publication in a future issue of this journal, but has not been fully edited.

Content may change prior to final publication in an issue of the journal. To cite the paper please use the doi provided on the Digital Library page.

for ease of interpretation, as shown in Fig. 9. It should be noted that the complete GA simulation took $131551 \mathrm{~s}$, which is roughly 37 hours; therefore each individual took, on average, approximately $42 \mathrm{~s}$ to evaluate all three objectives.

Visual interpretation of the 3D cloud of points in Fig. 9 is aided by the projected pareto fronts on each of the three planes. One such pareto front projection on the torqueefficiency plane of Fig. 9 is shown as Fig. 10 for clarity.

The concave pareto front on the torque-efficiency plane can be clearly seen as it is essentially continuous. Thus it can be deduced that the machine design trade-off between efficiency and torque must be made - as is always the case. That is, a higher torque will inevitably imply lower efficiency, and vice versa. The concavity of the pareto front on the torque-(power $\times$ speed) plane of Fig. 9 is less pronounced, which is largely due to the discontinuities of the design points on that particular pareto front. However, it is still possible to observe that the projected red plus markers onto the plane roughly indicate concavity; this implies that a high torque machine necessitates a lower constant power $\times$ speed product compared to a low torque machine. The pareto front could have resulted in a much more coherent and continuous front if either the number of individuals or the elitism score, or both, of the GA were set to a higher number [21]. The usual technique of obtaining a well-defined pareto front with the GA based optimization is of course through trial and error of the mentioned parameters, but this was not the main goal of this exercise. Furthermore, the current optimization task was to explore possible improvements upon the earlier machine design as reported in [4]. Finally, the pareto front on the efficiency(power $\times$ speed) plane is almost linear and has little effect on the efficiency of the machine, as seen in Fig. 9. This was to be expected since the efficiency estimation was for the fixed base-speed of the machine and was thus a function of the torque only.

For the computing time comparison it was recorded that the FEM simulations of the representative machine geometries took approximately 140 s each to complete taking into account the parallel speedup of two computing cores. Assuming less than ideal parallel speedup of only 1.8 times, rather than 2.0, the average FEM solution time was approximately $252 \mathrm{~s}$ - thus achieving at least six times the speedup with the flux tube based method compared to the FEM based solution. The speedup result is consistent with the previously undertaken flux tubes based GA optimization [15]. Further advantage of using flux tubes based optimization is that the requirement for extensive computing software and hardware is minimal as the method can be implemented with any modern computer scripting language such as Octave [22].

Returning to the point made in Section 2 regarding the accuracy of the flux tubes based SR machine analysis, it is now necessary to enquire if the same statement is true for the design optimization as well, as analysis and design are not the same tasks. The errors stemming from the flux tube based optimization for the torque, efficiency and rms current are displayed in Fig. 11 for several arbitrarily selected designs from Fig. 9 (red circles). The selection of points was random so as not to favour any particular design - it is a simple test to estimate average errors of the flux tube based method. As can be seen the discrepancies between the flux tube estimates and 2D FEM solutions are not large. It emerges that the averaged errors for the selected number of designs are $11 \%, 2 \%$ and less than $1 \%$ for the torque, current and efficiency, respectively.

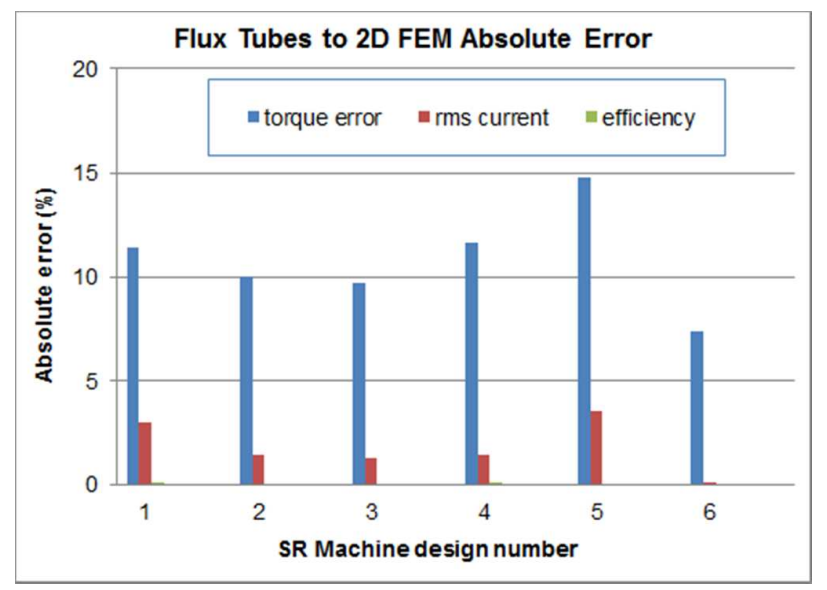

Fig. 11. Flux tubes method absolute error (\%) of torque, efficiency and rms current for the randomly selected SR machine designs from Fig. 9.

It can be argued therefore that the flux tubes based optimization furnishes objective functions which are accurate for the purpose of rapid initial design optimization of SR machines. It is reiterated here that the flux tubes accuracy can be controlled, and therefore increased, by selecting a larger number of flux tube slices $n$, while there were only $n=15$ such slices in Figs. 3 and $6-$ a very course subdivision compared to a fine subdivision in the case of FEM solutions.

\section{FLUX TUBE BASED DESIGN OPTIMIZATION CYCLE}

We can now revisit the concept of the hierarchical design, as depicted in Fig. 1 of Section 1, in the context of the SR machine design and the proposed flux tube approach. The basic steps in the original hierarchy in Fig. 1 will normally be followed as there is little choice in the provision of magnetic analysis techniques that are fast, intuitive and accurate at the same time. Thus in order to fully explore the available machine design space within acceptable computing times the designer is forced to follow the specified sequence. The flux tube approach, however, offers new opportunities due to its numerical error robustness and fast computations. The overall flux tube accuracy is not sufficient for the method to completely replace the more precise but time consuming 2D FEM, but does allow for the notion of rapid design to be introduced. This is elaborated upon in the flowchart of Fig. 12; it should be noted, however, that this flowchart describes a proposed design cycle, rather than suggesting a particular optimization algorithm. The advocated cycle defines a new paradigm in the rapid design of SR machines. 
This article has been accepted for publication in a future issue of this journal, but has not been fully edited. Content may change prior to final publication in an issue of the journal. To cite the paper please use the doi provided on the Digital Library page.

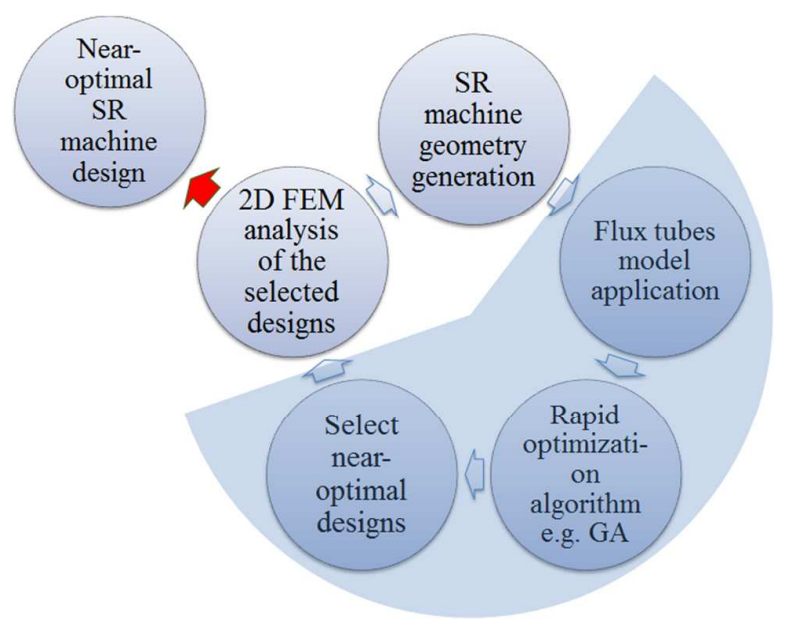

Fig. 12. Proposed rapid SR machine design cycle incorporating the flux tubes based modelling.

The rapid design process in the proposed cycle starts with the definition of the geometry of the SR machine and creation of the flux tube model (steps described in Section 2). After the flux tubes have been generated the model can be combined with a rapid optimization algorithm - as indicated by the third step in Fig. 12. Once the optimization based on the flux tube method has been completed the nearoptimal designs are chosen, those which appear the closest to the design goals for a particular application - this is step four in the design cycle. The selected designs, arrived at by way of flux tube model optimization, are reanalysed with an order of magnitude more accurate method (but at the same time much slower and non-intuitive) such as 2D FEM. If the FEM solutions confirm that the selected flux tube based designs are indeed near-optimal, the rapid design optimization cycle can conclude, as the near-optimal SR machine design is found and confirmed - this is the final step of the design cycle. However, if the 2D FEM indicates too large an error of all the selected designs, then the whole design cycle can be repeated in an iterative fashion until a satisfactory accuracy has been accomplished.

The proposed SR machine rapid design optimization cycle of Fig. 12 can now be put into perspective in relation to the hierarchical design of Fig. 1. The shaded segment in Fig. 12, which encompasses the three distinct design steps, can now - with its favourable qualities of fast and intuitive computations - largely replace the middle stage in the hierarchical design pyramid in Fig. 1, that is essentially replace the slow and non-intuitive 2D FEM solutions, since the numerical accuracy of the flux tube method was shown to be sufficient to guide the rapid initial optimization task to its successful completion. The same statement is emphasized in the proposed design cycle in Fig. 12, where the 2D FEM is not completely excluded, but is used sparsely. Another way of describing the process would be to say that the flux tube method complements the FEM by taking over the main burden of the computational effort. Thus the synergy of the various design methods and approaches is enhanced through appropriate combination of very fast and very accurate methods. Ultimately significant savings in computing resources and reduced computing times may be achieved at the most crucial stage of the SR machine design process, where the design space is still very large. Although the FEM computations are not completely eliminated, they are drastically reduced.

\section{Conclusions}

The following conclusions can be drawn regarding the rapid multi-objective optimization of SR motors assisted by the flux tubes modelling approach. First, as has been demonstrated by an example, the design cycle can be significantly shortened while preserving the original large design space. As a result substantial savings in computing resources and simulation times can be accomplished without loss of accuracy and avoiding the risk of missing the global optimum. Finally, the machine designer is presented with a fast, intuitive and practical optimization tool - one which can be utilized without elaborate computational resources.

Future implementations of the proposed flux tube based method are likely to include more elaborate representation of the flux tubes to model the leakage flux, as well as the design of synchronous reluctance machines where, similarly to the SR machines, the challenges of time consuming nonlinear magnetic simulations hinder the design process.

\section{Acknowledgments}

This work is kindly supported by an EPSRC grant (EP/G03690x/1).

\section{References}

[1] T. J. E. Miller, Switched Reluctance Motors and Their Control. Oxford University Press, Oxford UK, 1993.

[2] T. J. E. Miller ed., Electronic Control of Switched Reluctance Machines. Newnes Power Engineering Series, 2001.

[3] R. Krishnan, Switched Reluctance Motor Drives: Modeling, Simulation, Analysis, Design, and Applications. CRC Press LLC, Boca Raton Florida, 2001.

[4] M. Takeno, A Chiba, N. Hoshi, S. Ogasawara, M. Takemoto, and M. Azizur Rahman, "Test results and torque improvement of the $50-\mathrm{kW}$ switched reluctance motor designed for hybrid electric vehicle," IEEE Trans. Industry Applications, vol. 48, pp. 1327-1334, August 2012.

[5] N. Schofield, S. A. Long, D. Howe, and M. McClelland, "Design of a switched reluctance machine for extended speed operation", IEEE Transactions On Industry Applications, vol. 45, no. 1, January/February 2009.

[6] J. D. Widmer, R. Martin, and M. Kimiabeigi, "Electric vehicle traction motors without rare earth magnets," Sustainable Materials and Technologies, 3, pp. 7-13, Elsevier, 2015.

[7] K. M. Rahman, B. Fahimi, G. Suresh, A. V. Rajarathnam, and M. Ehsani, "Advantages of switched reluctance motor applications to EV and HEV: design and control issues," IEEE Trans. on Industry Applications, vol. 36, no. 1, January/February 2000.

[8] J. M. Stephenson and J. Corda, "Computation of torque and current in doubly salient reluctance motors from nonlinear magnetisation data", IEE Proc., vol. 126, no. 5, May 1979. 
This article has been accepted for publication in a future issue of this journal, but has not been fully edited.

Content may change prior to final publication in an issue of the journal. To cite the paper please use the doi provided on the Digital Library page.

[9] R. Krishnan, R. Arumugam, and J. F. Lindsay, "Design procedure for switched reluctance motors," IEEE Trans. on Industry Applications, vol. 24, no. 3, pp. 456-461, June 1988.

[10] T. J. E. Miller, "Nonlinear theory of the switched reluctance motor for rapid computer-aided design," IEE Proc., vol. 137, no. 6, Nov. 1990.

[11] M. Balaji and V. Kamaraj,"Evolutionary computation based multi-objective pole shape optimization of switched reluctance machine", Electrical Power and Energy Systems vol. 43 pp. 63-69, Elsvier, 2012.

[12] C. Ma and L. Qu, "Multiobjective Optimization of Switched Reluctance Motors Based on Design of Experiments and Particle Swarm Optimization," Paper 294, DigitalCommons@University of Nebraska Lincoln, 2015.

[13] Y. K. Choi, H. S. Yoon, and C. S. Koh, "Pole-shape optimization of a switched-reluctance motor for torque ripple reduction," IEEE Trans. Magn., vol. 43, no. 4, pp. 1797-1800, 2007.

[14] A. Stuikys, M. Rotaru, and J. K. Sykulski, "A refined approach exploiting tubes of flux for analysis of linear switched reluctance motor," International Journal of Applied Electromagnetics and Mechanics, vol. 51, pp. S13-S21, IOS Press, 2016.

[15]A. Stuikys and J. K. Sykulski, "An efficient design optimization framework for nonlinear switched reluctance machines," IEEE Trans. Industry Applications, vol. 53, no. 3, pp. 1985-1993, February 2017.

[16]H. C. Roters, Electromagnetic Devices, John Wiley \& Sons Inc., 1941.

[17] L. Byeong-Seok, "Linear switched reluctance machine drives with electromagnetic levitation and guidance systems," Doctoral Thesis, Virginia Polytechnic Institute and State University, November, 2000.

[18] U. S. Deshpande, J. J. Cathey, and E. Richter, "Highforce density linear switched reluctance machine," IEEE Transactions On Industry Applications, vol. 31, pp. 345-352, no. 2, March/April 1995.

[19] C. A. Vaithilingam, N. Misron, M. R. Zare, I. Aris, and M. H. Marhaban, "Computation of electromagnetic torque in a double rotor switched reluctance motor using flux tube methods," Energies, 5, pp. 4008-4026, DOI:10.3390/en5104008, 2012.

[20] J. K. Sykulski, "Dual field modeling using tubes and slices," Advances in Engineering Software, vol. 21, pp. 21-26, Elsevier Science Limited, 1995.

[21]D. E. Goldberg, Genetic Algorithms in Search, Optimization \& Machine Learning. Addison-Wesley, 1989.

[22] GNU Octave, https://www.gnu.org/software/octave/ , accessed 10 April 2017. 